\title{
Multiple atrial premature contractions predict stroke recurrence in patients with cryptogenic stroke and concomitant non-sustained atrial tachycardia
}

\author{
Jung-Joon Cha' ${ }^{1}$ Hyemoon Chung ${ }^{2}$, Jae-Sun Uhm ${ }^{1}$, Boyoung Joung ${ }^{1}$, Hui-Nam Pak' ${ }^{1}$ Moon-Hyoung Lee ${ }^{1}$
} and Jong-Youn $\mathrm{Kim}^{3^{*}}$

\begin{abstract}
Background and objectives: Atrial fibrillation is a known risk factor for cryptogenic stroke and therefore requires early detection and prompt management. We investigated predictors of stroke recurrence in patients with cryptogenic stroke and concomitant non-sustained atrial tachycardia but not atrial fibrillation.

Subjects and methods: We investigated 390 patients (219 men, mean age $67 \pm 12$ years) diagnosed with stroke and non-sustained atrial tachycardia on 24-h Holter monitoring and recorded the total number of atrial premature contractions identified by the Holter monitor. Multiple atrial premature contractions were defined as atrial premature contractions $>34$ beats/day. We analyzed the rates of 5-year freedom from stroke recurrence or atrial fibrillation and investigated independent predictors of stroke recurrence and undiagnosed atrial fibrillation.

Results: The mean follow-up period was $35 \pm 21$ months, and the overall stroke recurrence rate was 9.0\%. KaplanMeier survival analysis revealed that the rate of freedom from stroke recurrence was significantly lower in patients with multiple atrial premature contractions. Also, the patients with multiple atrial premature contractions had higher cumulative incidence rate of new-onset $\operatorname{AF}(p=0.019)$. Multivariate analysis showed that multiple atrial premature contractions (hazard ratio 2.49,95\% confidence interval 1.05-5.88, $p=0.038$ ), cigarette smoking status (hazard ratio $2.66,95 \%$ confidence interval 1.15-6.17, $p=0.022$ ), and the left atrial volume index (hazard ratio $1.05,95 \%$ confidence interval 1.01-1.09, $p=0.020$ ) were significantly associated with stroke recurrence in patients with cryptogenic stroke. However, these factors were not statistically significant predictors of future onset of atrial fibrillation.

Conclusions: Multiple atrial premature contractions were significantly correlated with an increased left atrial volume index, which could predict future onset of atrial fibrillation and stroke recurrence. This study showed that multiple atrial premature contractions predict stroke recurrence in patients with cryptogenic stroke without atrial fibrillation.
\end{abstract}

Keywords: Cryptogenic stroke, Stroke recurrence, Atrial fibrillation, Atrial premature contraction

\footnotetext{
*Correspondence: jykim0706@yuhs.ac

${ }^{3}$ Division of Cardiology, Department of Internal Medicine, Gangnam Severance Hospital, Yonsei University College of Medicine, 146-92, Dogok-dong, Gangnam-gu, Seoul 06273, Korea

Full list of author information is available at the end of the article
}

\author{
Introduction \\ Atrial fibrillation (AF) is a well-known risk factor for cer- \\ ebral infarction; however, AF is often diagnostically chal- \\ lenging owing to its paroxysmal or asymptomatic clinical \\ presentation [1-3]. A Holter electrocardiogram (ECG) \\ is a useful diagnostic tool for AF; however, low diagnos- \\ tic yield is a limitation of this modality [2, 4]. Although
}

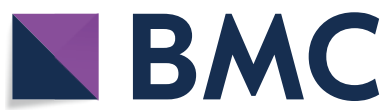

(c) The Author(s) 2020. This article is licensed under a Creative Commons Attribution 4.0 International License, which permits use, sharing, adaptation, distribution and reproduction in any medium or format, as long as you give appropriate credit to the original author(s) and the source, provide a link to the Creative Commons licence, and indicate if changes were made. The images or other third party material in this article are included in the article's Creative Commons licence, unless indicated otherwise in a credit line to the material. If material is not included in the article's Creative Commons licence and your intended use is not permitted by statutory regulation or exceeds the permitted use, you will need to obtain permission directly from the copyright holder. To view a copy of this licence, visit http://creativecommons.org/licenses/by/4.0/. 
a loop recorder is a valuable diagnostic aid for AF [5], invasiveness and high costs are limitations precluding its routine use.

The clinical presentation and sequelae of cerebral infarction are more severe in patients with AF; therefore, early detection of AF significantly improves patients' prognosis and reduces overall health care costs [6]. Previous studies have reported that atrial premature contractions (APCs) and non-sustained atrial tachycardia (NSAT) observed on Holter ECG may predict undiagnosed AF $[7,8]$. We investigated the predictors of stroke recurrence in patients with cryptogenic stroke and concomitant NSAT but not AF.

\section{Methods \\ Patients}

We investigated 2091 patients diagnosed with acute ischemic stroke without intracardiac thrombi. All patients underwent 24-h Holter monitoring, and of these 2091 patients, 390 with NSAT were included in the study. A total of 390 consecutive patients admitted to three tertiary hospitals (Severance Hospital, Gangnam Severance Hospital, and Kyung Hee University Hospital) between January 2009 and December 2012. All patients were diagnosed with the first episode of acute ischemic stroke on the current admission, along with NSAT on 24-h Holter monitoring, but without a diagnosis of AF before the present stroke. Brain magnetic resonance imaging and magnetic resonance angiography were performed to exclude atherosclerotic or cardioembolic strokes and structural heart diseases. Additionally, we excluded patients with intracardiac thrombi observed on transesophageal echocardiography. Namely, in TOAST classification [9], etiologies of the enrolled patients were included small vessel disease, other determined etiology and undetermined etiology.

All enrolled patients underwent transthoracic echocardiography (TTE), transesophageal echocardiography, and 24-h Holter monitoring within 7 days of admission. NSAT was defined as episodes of $\geq 3$ consecutive APCs with a heart rate $>100$ beats/min lasting $<30 \mathrm{~s}$. An APC was defined based on the following electrocardiographic parameters: a shortened RR interval $(\geq 25 \%$ reduction in interval duration), the occurrence of a $\mathrm{P}$ wave, and QRS width $<0.12 \mathrm{~s}$. The definition of the MAPC burden as frequency APCs had been varied according to their individual study results [10-12]. To determine the best cutoff values, which has the best performance of both sensitivity and specificity for predictors of stroke recurrence, receiver operating characteristic analysis was performed using the Youden index (sensitivity + specificity -1$)$. Based on the area under the curve value $(0.592)$, APCs $>34$ beats/day were defined as multiple APCs
(MAPCs) (sensitivity 77\%, specificity 44\%). Stroke was diagnosed based on the International Classification of Diseases, 9th Revision. Patients underwent computed tomography or magnetic resonance imaging for neurological evaluation. Exclusion criteria were as follows: (1) a history of stroke or transient ischemic attack, (2) known tachyarrhythmia, including supraventricular tachyarrhythmia, (3) atherosclerotic plaques involving the aorta or the aortic arch and the carotid artery, (4) structural heart disease, (5) permanent pacemaker implantation, (6) a history of coronary artery disease, brain hemorrhage, brain tumor, and cerebrovascular malformations, (7) uncontrolled hypertension, (8) reduced left ventricular (LV) ejection fraction (EF) $(<50 \%)$, and (9) neoplastic conditions. Patients were categorized into those without stroke recurrence $(n=355)$ and those with stroke recurrence $(n=35)$. Patients' electronic medical records were reviewed, and the relevant data were recorded. The study protocol complied with the Declaration of Helsinki and was approved by the Institutional Review Board of the Severance Cardiovascular Hospital, Seoul, Korea. Written informed consent was obtained from all patients included in the study.

\section{Echocardiographic evaluation}

All patients underwent comprehensive Doppler echocardiography based on the current American Society of Echocardiography guidelines. The peak early $(E)$ and late diastolic mitral inflow velocities were measured, and tissue Doppler imaging from the septal aspect of the mitral annulus was performed to measure the early diastolic mitral annulus peak velocity $(e ')$ in an apical fourchamber view. The $E / e^{\prime}$ ratio was calculated based on these values, and the EF was calculated using the modified Simpson rule. The left atrial (LA) anterior-posterior diameter was measured in a parasternal long-axis view at end systole to determine the LA size. The ellipse method was used to measure the LA volume from apical fourchamber and parasternal long-axis views at ventricular end systole.

The effect of body surface area on LA volume was adjusted using an indexed value that was calculated by dividing the LA volume by body surface area (LA volume index [LAVi]).

\section{Management and follow-up}

For the first diagnosis of ischemic stroke, all patients underwent standard ECG, 24-h Holter monitoring, and echocardiography to identify cardioembolic sources of stroke. Antiplatelet agents (aspirin, clopidogrel, or triflusal) were prescribed after excluding a cardioembolic stroke. Stroke recurrence was defined as the occurrence 
of neurological symptoms corroborated by objective evidence of lesions on brain imaging.

\section{Statistical analyses}

Continuous and categorical variables were expressed as mean \pm standard deviation and counts (percentages), respectively. Baseline characteristics were compared between patients with and without stroke recurrence using the Student $t$ test for continuous variables and the Chi-square test for categorical variables. A $p$ value $<0.05$ was considered statistically significant. We analyzed receiver operating characteristic curves with the Youden index to determine the optimal cutoff value for stroke recurrence. Stroke recurrence rates were compared between patients with and without MAPCs using the 5-year Kaplan-Meier survival curves with the log-rank test. Cox proportional hazards regression analysis was performed using univariate and multivariate models to determine independent predictors of stroke recurrence in patients with NSAT. Variables that showed a $p$ value $<0.10$ in univariate analysis or those considered relevant predictors of clinical events were subjected to multivariate analysis. All statistical analyses were performed using the SPSS software (IBM, Markham, Canada).

\section{Results}

The mean age of the 390 patients included in this study was 67 years. Of these 390 patients, the mean age of patients with and without stroke recurrence was 69 and 67 years, respectively. No statistically significant intergroup difference was observed in mean age $(p=0.239)$. No statistically significant intergroup difference was observed in patients' baseline clinical characteristics. However, with regard to echocardiographic parameters, the LAVi was higher in patients with stroke recurrence than in those without stroke recurrence ( 28 vs. 24 , $p=0.005$ ) (Table 1).

The mean follow-up period was $35 \pm 21$ months, and the overall stroke recurrence rate was $9.0 \%$ (35 of 390 patients). Kaplan-Meier survival analysis revealed that the rate of freedom from stroke recurrence was significantly lower in patients with MAPCs than in those without MAPCs $(p=0.008)$ (Fig. 1$)$.

Table 1 Patient characteristics

\begin{tabular}{|c|c|c|c|c|}
\hline & \multirow[t]{2}{*}{ All } & \multicolumn{2}{|c|}{ Stroke Recurrence } & \multirow[t]{2}{*}{$p$} \\
\hline & & No & Yes & \\
\hline Number of patients & 390 & 355 & 35 & \\
\hline Age (years) & $66.7 \pm 11.9$ & $66.5 \pm 11.8$ & $69.0 \pm 12.7$ & 0.239 \\
\hline Male sex, $n(\%)$ & $219(56.2 \%)$ & $200(56.3 \%)$ & $19(54.3 \%)$ & 0.815 \\
\hline Hypertension, $n(\%)$ & $273(70.0 \%)$ & $246(69.3 \%)$ & $27(77.1 \%)$ & 0.440 \\
\hline Diabetes, $n(\%)$ & $107(27.4 \%)$ & $102(28.7 \%)$ & $5(14.3 \%)$ & 0.075 \\
\hline Dyslipidemia, $n$ (\%) & $160(41.0 \%)$ & $145(40.8 \%)$ & $15(42.9 \%)$ & 0.858 \\
\hline Chronic kidney disease, $n$ (\%) & $17(4.4 \%)$ & $14(3.9 \%)$ & $3(8.6 \%)$ & 0.383 \\
\hline Vascular disease, $n(\%)$ & $12(3.1 \%)$ & $10(2.8 \%)$ & $2(5.7 \%)$ & 0.294 \\
\hline Coronary artery disease, $n(\%)$ & $41(10.5 \%)$ & $37(10.4 \%)$ & $4(11.4 \%)$ & 0.775 \\
\hline Cigarette smokers, n (\%) & $148(37.9 \%)$ & $130(36.6 \%)$ & $18(51.4 \%)$ & 0.101 \\
\hline \multicolumn{5}{|l|}{ Echocardiographic parameters } \\
\hline LA diameter $(\mathrm{mm})$ & $36.4 \pm 4.5$ & $36.3 \pm 4.5$ & $37.1 \pm 4.7$ & 0.353 \\
\hline LA volume $\left(\mathrm{mm}^{3}\right)$ & $41.2 \pm 11.8$ & $40.8 \pm 11.6$ & $45.6 \pm 13.1$ & 0.020 \\
\hline LA volume index $\left(\mathrm{mm}^{3} / \mathrm{m}^{2}\right)$ & $24.5 \pm 6.9$ & $24.2 \pm 6.6$ & $27.7 \pm 9.0$ & 0.005 \\
\hline LVEF (\%) & $66.6 \pm 8.8$ & $66.7 \pm 8.8$ & $66.1 \pm 8.3$ & 0.707 \\
\hline LV mass index $\left(\mathrm{g} / \mathrm{m}^{2}\right)$ & $106.1 \pm 28.9$ & $105.5 \pm 27.3$ & $111.9 \pm 42.6$ & 0.231 \\
\hline$E / e^{\prime}$ & $12.0 \pm 4.5$ & $11.9 \pm 4.4$ & $12.9 \pm 5.0$ & 0.206 \\
\hline $\operatorname{APCs}(n)$ & $541.0 \pm 2565.7$ & $528.6 \pm 2605.4$ & $636.3 \pm 2262.9$ & 0.791 \\
\hline MAPCs & $229(58.7 \%)$ & $202(56.9 \%)$ & $27(77.1 \%)$ & 0.030 \\
\hline \multicolumn{5}{|l|}{ Medication } \\
\hline Aspirin, $n(\%)$ & $301(77.2 \%)$ & $273(76.9 \%)$ & $28(80.0 \%)$ & 0.834 \\
\hline Triflusal, $n(\%)$ & $30(7.7 \%)$ & $29(8.2 \%)$ & $1(2.9 \%)$ & 0.501 \\
\hline Clopidogrel, $n(\%)$ & $203(52.1 \%)$ & $185(52.1 \%)$ & $18(51.4 \%)$ & 1.000 \\
\hline
\end{tabular}

$A P C s$ atrial premature contractions, $E / e^{\prime}$ ratio of the peak early diastolic mitral inflow velocity to early diastolic mitral annulus peak velocity, $L A$ left atrium, $L V$ left ventricular, $L V E F$ left ventricular ejection fraction, $M A P C s$ multiple atrial premature contractions 


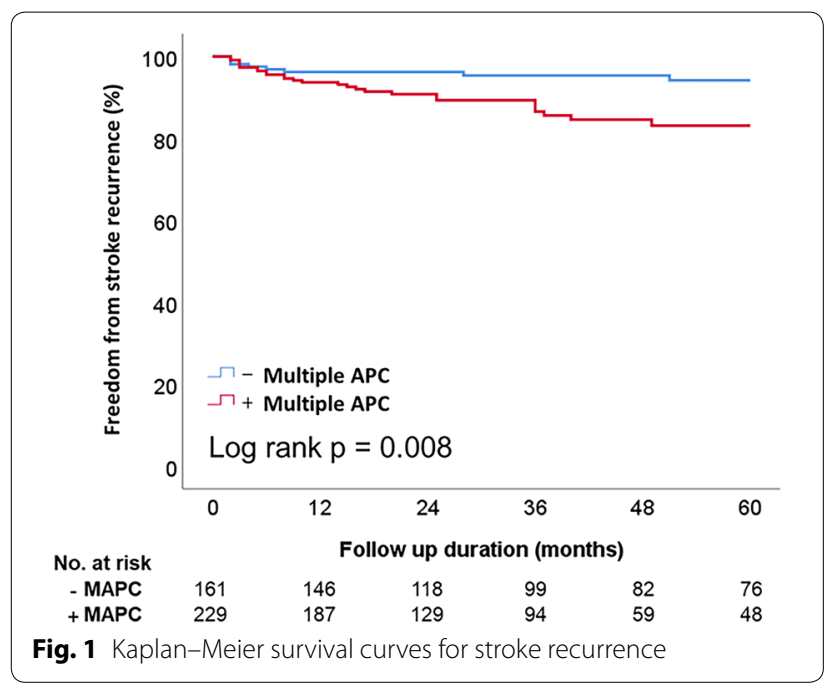

Cox proportional hazard regression analysis was performed using univariate and multivariate models to investigate the predictors of stroke recurrence in patients with cryptogenic stroke (Table 2). Univariate analysis showed that the LAVi (hazards ratio [HR] 1.06, 95\% confidence interval [CI] 1.02-1.10, $p=0.004)$ and MAPCs (HR 2.80, 95\% CI 1.27-6.17, $p=0.011$ ) were predictors of stroke recurrence in patients with cryptogenic stroke. Multivariate analysis after adjusting for confounders, such as age, sex, hypertension, diabetes, cigarette smoking status, and LAVi, showed that MAPCs (HR 2.49, 95\% CI 1.05-5.88, $p=0.038)$, cigarette smoking status (HR 2.66, 95\% CI 1.15-6.17, $p=0.022$ ), and the LAVi (HR 1.05, 95\% CI 1.01-1.09, $p=0.020$ ) were significantly associated with stroke recurrence in patients with cryptogenic stroke and concomitant NSAT.

Prevalence rates of new-onset AF were 10 of 229 (4.4\%) and 2 of $161(1.2 \%)$ in patients with and without MAPCs, respectively. Figure 2 shows the Kaplan-Meier survival curves for new-onset AF in both groups. The cumulative

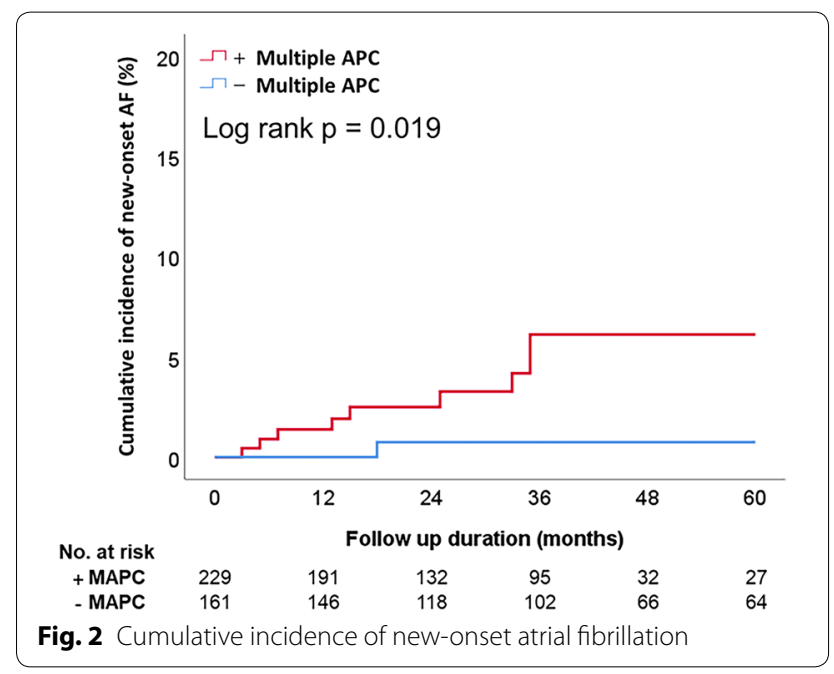

incidence rate of new-onset AF was significantly higher in patients with MAPCs $(p=0.019)$. Univariate Cox proportional hazards regression analysis showed that MAPCs (HR 5.29, 95\% CI 1.13-24.71, $p=0.034$ ), age (HR $1.06,95 \%$ CI 1.00-1.12, $p=0.046)$, chronic kidney disease (HR 5.64, 95\% CI 1.23-25.96, $p=0.026$ ), LAVi (HR 1.08, 95\% CI 1.01-1.15, $p=0.007)$, and $E / e^{\prime}$ (HR 1.01, 95\% CI 1.04-1.27, $p=0.007)$ were significant predictors of newonset AF (Table 3). However, these variables were statistically nonsignificant when subjected to multivariate Cox proportional hazards regression analysis.

\section{Discussion}

This study highlights that MAPCs and an increased LAVi were significantly associated with stroke recurrence in patients with cryptogenic stroke and concomitant NSAT. Based on the findings of our study, we recommend that patients with cryptogenic stroke who

Table 2 Cox proportional hazards regression analysis of predictors of stroke recurrence

\begin{tabular}{|c|c|c|c|c|c|c|}
\hline & \multicolumn{3}{|c|}{ Univariate } & \multicolumn{3}{|c|}{ Multivariate } \\
\hline & HR & $95 \% \mathrm{Cl}$ & $p$ & HR & $95 \% \mathrm{Cl}$ & $p$ \\
\hline Age (years) & 1.02 & $0.99-1.05$ & 0.190 & 1.00 & $0.97-1.04$ & 0.908 \\
\hline Male sex, $n(\%)$ & 1.11 & $0.57-2.16$ & 0.757 & 0.63 & $0.27-1.46$ & 0.282 \\
\hline Hypertension, n (\%) & 1.44 & $0.66-3.17$ & 0.364 & 1.28 & $0.57-2.89$ & 0.558 \\
\hline Diabetes, $n(\%)$ & 0.41 & $0.16-1.06$ & 0.065 & 0.41 & $0.16-1.06$ & 0.065 \\
\hline Cigarette smokers, $n(\%)$ & 1.78 & $0.92-3.45$ & 0.089 & 2.66 & $1.15-6.17$ & 0.022 \\
\hline LA volume index $\left(\mathrm{mm}^{3} / \mathrm{m}^{2}\right)$ & 1.06 & $1.02-1.10$ & 0.004 & 1.05 & $1.01-1.09$ & 0.020 \\
\hline MAPCs & 2.79 & $1.27-6.17$ & 0.011 & 2.49 & $1.05-5.88$ & 0.038 \\
\hline
\end{tabular}

$C l$ confidence interval, $H R$ hazard ratio, $L A$ left atrium, $M A P C s$ multiple atrial premature contractions 
Table 3 Cox proportional hazards regression analysis of predictors of new-onset atrial fibrillation

\begin{tabular}{|c|c|c|c|c|c|c|}
\hline & \multicolumn{3}{|c|}{ Univariate } & \multicolumn{3}{|c|}{ Multivariate } \\
\hline & HR & $95 \% \mathrm{Cl}$ & $p$ & HR & $95 \% \mathrm{Cl}$ & $p$ \\
\hline Age (years) & 1.06 & $1.00-1.12$ & 0.046 & 1.02 & $0.96-1.09$ & 0.461 \\
\hline Chronic kidney disease, $n$ (\%) & 5.64 & $1.23-25.96$ & 0.026 & 2.42 & $0.47-12.37$ & 0.289 \\
\hline LA volume index $\left(\mathrm{mm}^{3} / \mathrm{m}^{2}\right)$ & 1.08 & $1.01-1.15$ & 0.020 & 1.04 & $0.97-1.12$ & 0.289 \\
\hline$E / e^{\prime}$ & 1.15 & $1.04-1.27$ & 0.007 & 1.07 & $0.96-1.20$ & 0.221 \\
\hline MAPCS & 5.29 & $1.13-24.71$ & 0.034 & 3.12 & $0.60-16.27$ & 0.177 \\
\hline
\end{tabular}

$C l$ confidence interval, $E / e^{\prime}$ ratio of the peak early diastolic mitral inflow velocity to early diastolic mitral annulus peak velocity, $H R$ hazard ratio, $L A$ left atrium, $M A P C S$ multiple atrial premature contractions

present with the aforementioned risk factors should undergo close and prolonged follow-up with cardiac rhythm monitoring for early detection of future AF to ensure improved clinical outcomes.

We observed that MAPCs were independent predictors of stroke recurrence in patients with NSAT without previously diagnosed AF. The definition of the MAPC burden remains unclear. Previous investigators have defined MAPCs as frequent APCs, based on their individual study results, which represents a subjective interpretation [10-12]. Nevertheless, compared to patients without frequent APCs, those with frequent APCs showed poorer clinical outcomes [10-12]. Reportedly, in addition to the APC burden detected by Holter monitoring, APCs observed even in a single ECG tracing can affect clinical outcomes [13]. Although studies have reported an association between APCs and stroke, the exact pathomechanism remains unclear. A possible explanation could be that APCs are known precursors of AF, which is a major risk factor for stroke $[8,14]$. Additionally, increased LA volume, which showed a statistically significant association with APCs, was a predictor of stroke recurrence in this study. LAVi is a well-known risk factor for cardioembolic stroke $[15,16]$. Reportedly, atrial stretch [17] and atrial tissue alterations [18] in an enlarged LA are associated with disturbances of impulse propagation in patients with AF, suggesting that LA enlargement may increase the atrial ectopy rate and consequently the stroke risk [19]. In addition, the association between smoking and stroke recurrence had statistically significant. Lots of factors including oxidative stress, endothelial dysfunction, and inflammation by smoking aggravates atherosclerosis, as a consequence, stroke occurrence [20,21]. Moreover, Chen et al. [22] lately reported that smoking increases the risk of stroke recurrence.

Previous studies have reported an association between APCs and AF [14, 23, 24] and proved that frequent APCs or consecutive supraventricular extrasystole, such as NSAT, were associated with a higher prevalence of AF [14, 23, 24]. In this study, the cumulative incidence rate of AF was statistically significant in patients with MAPCs; this finding concurs with that of the aforementioned studies. Cox proportional hazards regression analysis using a univariate model confirmed the association between MAPCs and AF. However, multivariate analysis did not show conclusive results. These observations could be attributed to the baseline differences in LA size between patients with and without AF. LA enlargement, which predicts cardiovascular events, is common in patients with AF [25]. A previous study reported that each $5-\mathrm{mm}$ increase in the LA diameter increased the risk of new-onset $\mathrm{AF}$ by $39 \%$ [26]. We previously reported that LAVi was an independent predictor of AF [27]. However, multivariate analysis performed in the present study showed that neither MAPCs nor the LAVi was a statistically significant predictor of future AF. Large-scale studies are warranted to further investigate this issue.

The following are the limitations of this study: (1) This retrospective study was performed across 3 tertiary referral hospitals. Therefore, a selection and referral bias cannot be completely excluded. (2) The relatively small size of the study population and lack of adequate data (including follow-up Holter ECG and additional echocardiographic parameters, such as 3D measurements) serve as drawbacks of this study. (3) The statistical model used may not have accurately analyzed unmeasured confounding factors, including patients' general health condition and $3 \mathrm{D}$ techniques used for TTE in the multivariate analysis.

In conclusion, MAPCs were significantly associated with an increased LAVi, which predicts stroke recurrence in patients with cryptogenic stroke and concomitant NSAT. This study highlights that the MAPC burden can be considered a useful predictor of stroke recurrence in patients with cryptogenic stroke and concomitant NSAT but not AF. 


\section{Abbreviations}

AF: Atrial fibrillation; APC: Atrial premature contraction; Cl: Confidence interval; ECG: Electrocardiogram; EF: Ejection fraction; HR: Hazard ratio; LA: Left atrial; LAVi: Left atrial volume index; LV: Left ventricular; MAPC: Multiple atrial premature contraction; NSAT: Non-sustained atrial tachycardia; TTE: Transthoracic echocardiography.

\section{Acknowledgements}

None.

\section{Authors' contributions}

J-JC involved in writing draft and analyzing data. J-YK involved in data and writing draft and creating concept of study. HC, J-SU, BJ, H-NP, and M-HL involved in data review and writing draft. All authors read and approved the final manuscript.

\section{Funding}

This study supported by a grant from the Korean Heart Rhythm Society (Grant Number KHRS2016-2)

\section{Availability of supporting data}

None.

\section{Ethical approval and consent to participate}

The study protocol complied with the Declaration of Helsinki and was approved by the Institutional Review Board of the Severance Cardiovascular Hospital, Seoul, Korea. Written informed consent was obtained from all patients included in the study.

\section{Consent for publication}

Not applicable.

\section{Competing interests}

The authors declare that they have no competing interests.

\section{Author details}

${ }^{1}$ Division of Cardiology, Department of Internal Medicine, Severance Hospital, Seoul, Korea. ${ }^{2}$ Department of Cardiology, College of Medicine, Kyung Hee University, Seoul, Korea. ${ }^{3}$ Division of Cardiology, Department of Internal Medicine, Gangnam Severance Hospital, Yonsei University College of Medicine, 146-92, Dogok-dong, Gangnam-gu, Seoul 06273, Korea

Received: 21 November 2019 Accepted: 14 April 2020

Published online: 21 April 2020

\section{References}

1. Lin HJ, Wolf PA, Kelly-Hayes M, Beiser AS, Kase CS, Benjamin EJ, et al. Stroke severity in atrial fibrillation. The Framingham study. Stroke. 1996:27(10):1760-4

2. Kirchhof P, Auricchio A, Bax J, Crijns H, Camm J, Diener H-C, et al. Outcome parameters for trials in atrial fibrillation: executive summary: recommendations from a consensus conference organized by the German Atrial Fibrillation Competence NETwork (AFNET) and the European Heart Rhythm Association (EHRA). Eur Heart J. 2007;28(22):2803-17.

3. Liao J, Khalid Z, Scallan C, Morillo C, O'Donnell M. Noninvasive cardiac monitoring for detecting paroxysmal atrial fibrillation or flutter after acute ischemic stroke: a systematic review. Stroke. 2007;38(11):2935-40.

4. Kessler DK, Kessler KM. Is ambulatory electrocardiography useful in the evaluation of patients with recent stroke? Chest. 1995;107(4):916-8.

5. Diederichsen SZ, Haugan KJ, Kober L, Hojberg S, Brandes A, Kronborg C, et al. Atrial fibrillation detected by continuous electrocardiographic monitoring using implantable loop recorder to prevent stroke in individuals at risk (the LOOP study): rationale and design of a large randomized controlled trial. Am Heart J. 2017:187:122-32.

6. Go AS, Hylek EM, Phillips KA, Chang Y, Henault LE, Selby JV, et al. Prevalence of diagnosed atrial fibrillation in adults: national implications for rhythm management and stroke prevention: the AnTicoagulation and Risk Factors in Atrial Fibrillation (ATRIA) Study. JAMA. 2001;285(18):2370-5.

7. Stahrenberg R, Weber-Kruger M, Seegers J, Edelmann F, Lahno R, Haase $B$, et al. Enhanced detection of paroxysmal atrial fibrillation by early and prolonged continuous Holter monitoring in patients with cerebral ischemia presenting in sinus rhythm. Stroke. 2010;41(12):2884-8.

8. Wallmann D, Tuller D, Kucher N, Fuhrer J, Arnold M, Delacretaz E. Frequent atrial premature contractions as a surrogate marker for paroxysmal atrial fibrillation in patients with acute ischaemic stroke. Heart. 2003;89(10):1247-8

9. Adams HP Jr, Bendixen BH, Kappelle L, Biller J, Love BB, Gordon DL, et al. Classification of subtype of acute ischemic stroke. Definitions for use in a multicenter clinical trial. TOAST. Trial of Org 10172 in Acute Stroke Treatment. Stroke. 1993;24(1):35-41.

10. Vinther KH, Tveskov C, Moller S, Auscher S, Osmanagic A, Egstrup K. Excessive premature atrial complexes and the risk of recurrent stroke or death in an ischemic stroke population. J Stroke Cerebrovasc Dis. 2017:26(6):1163-70.

11. Larsen BS, Kumarathurai P, Falkenberg J, Nielsen OW, Sajadieh A. Excessive atrial ectopy and short atrial runs increase the risk of stroke beyond incident atrial fibrillation. J Am Coll Cardiol. 2015;66(3):232-41.

12. Binici Z, Intzilakis T, Nielsen OW, Kober L, Sajadieh A. Excessive supraventricular ectopic activity and increased risk of atrial fibrillation and stroke. Circulation. 2010:121(17):1904-11.

13. Inohara T, Kohsaka S, Okamura T, Watanabe M, Nakamura Y, Higashiyama $\mathrm{A}$, et al. Long-term outcome of healthy participants with atrial premature complex: a 15-year follow-up of the NIPPON DATA 90 Cohort. PLOS ONE. 2013;8(11):e80853.

14. Kochhauser S, Dechering DG, Dittrich R, Reinke F, Ritter MA, Ramtin S, et al Supraventricular premature beats and short atrial runs predict atrial fibrillation in continuously monitored patients with cryptogenic stroke. Stroke. 2014;45(3):884-6

15. Fatema K, Bailey KR, Petty GW, Meissner I, Osranek M, Alsaileek AA, et al. Increased left atrial volume index: potent biomarker for first-ever ischemic stroke. Mayo Clin Proc. 2008;83(10):1107-14.

16. Di Tullio MR, Sacco RL, Sciacca RR, Homma S. Left atrial size and the risk of ischemic stroke in an ethnically mixed population. Stroke. 1999;30(10):2019-24.

17. Satoh T, Zipes DP. Unequal atrial stretch in dogs increases dispersion of refractoriness conducive to developing atrial fibrillation. J Cardiovasc Electrophysiol. 1996;7(9):833-42.

18. Keren G, Etzion T, Sherez J, Zelcer AA, Megidish R, Miller HI, et al. Atrial fibrillation and atrial enlargement in patients with mitral stenosis. Am Heart J. 1987;114(5):1146-55.

19. Marcus GM, Dewland TA. Premature atrial contractions: a wolf in sheep's clothing? J Am Coll Cardiol. 2015:66(3):242-4

20. Ambrose JA, Barua RS. The pathophysiology of cigarette smoking and cardiovascular disease: an update. J Am Coll Cardiol. 2004;43(10):1731-7.

21. Wolf PA, D'Agostino RB, Kannel WB, Bonita R, Belanger AJ. Cigarette smoking as a risk factor for stroke: the Framingham Study. JAMA. 1988;259(7):1025-9.

22. Chen J, Li S, Zheng K, Wang H, Xie Y, Xu P, et al. Impact of smoking status on stroke recurrence. J Am Heart Assoc. 2019;8(8):e011696.

23. Gladstone DJ, Dorian P, Spring M, Panzov V, Mamdani M, Healey JS, et al. Atrial premature beats predict atrial fibrillation in cryptogenic stroke: results from the EMBRACE trial. Stroke. 2015:46(4):936-41.

24. Chong B-H, Pong V, Lam K-F, Liu S, Zuo M-L, Lau Y-F, et al. Frequent premature atrial complexes predict new occurrence of atrial fibrillation and adverse cardiovascular events. EP Eur. 2011;14(7):942-7.

25. Mochizuki A, Yuda S, Oi Y, Kawamukai M, Nishida J, Kouzu H, et al. Assessment of left atrial deformation and synchrony by three-dimensional speckle-tracking echocardiography: comparative studies in healthy subjects and patients with atrial fibrillation. J Am Soc Echocardiogr. 2013;26(2):165-74.

26. Vaziri SM, Larson MG, Benjamin EJ, Levy D. Echocardiographic predictors of nonrheumatic atrial fibrillation. The Framingham Heart Study. Circulation. 1994:89(2):724-30.

27. Chung H, Joung B, Lee KY, Uhm JS, Pak HN, Lee MH, et al. Left atrial volume index predicts recurrence of stroke in patients with nonsustained atrial tachycardia. J Stroke Cerebrovasc Dis. 2015;24(10):2408-15.

\section{Publisher's Note}

Springer Nature remains neutral with regard to jurisdictional claims in published maps and institutional affiliations. 